\title{
Contracted Endodontic Cavity in a Severely Calcified Anterior Tooth Using Guided Endodontics Technique: Case Report
}

\author{
Alessandra Rosa de Sá ${ }^{1}$, José Flávio Gabrich Giovannini ${ }^{1}$, Henrique \\ Trindade de Magalhães ${ }^{1}$, Victor Couto Figueiredo ${ }^{2}$, Tiago Braga $^{3}$ and \\ Rodrigo Rodrigues Amaral ${ }^{2,3 *}$ \\ ${ }^{1}$ Department of Dentistry, Centro Universitário Newton Paiva, Faculdade de Odonto- \\ logia, Belo Horizonte, Minas Gerais, Brazil \\ ${ }^{2}$ Department of Dentistry, Pontifícia Universidade Católica de Minas Gerais, Belo \\ Horizonte, Minas Gerais, Brazil \\ ${ }^{3}$ School of Dentistry and Oral Health Griffith University, Gold Coast, Queensland, \\ Australia \\ *Corresponding Author: Rodrigo Rodrigues Amaral, School of Dentistry and \\ Oral Health, Griffith University, Gold Coast Campus, Queensland, Australia. \\ DOI: $10.31080 /$ ASDS.2020.04.0896
}

\author{
Received: June 29, 2020 \\ Published: July 28, 2020 \\ (C) All rights are reserved by Evanthia \\ Chrysomali., et al.
}

\begin{abstract}
Pulp canal obliteration (PCO) is characterized by the deposition of calcified tissue within the root canal space, which may partially or totally involve the canal. The endodontic treatment of calcified teeth is considered a challenging task depending on the severity of pulp obliteration. Recently, guided endodontics has become an alternative approach for those cases. This case report describes the use of guided endodontic access for the maxillary left lateral incisor of a 73-year-old male patient that required restorative treatment. The tooth had an extensive coronary fracture and demanded endodontic treatment for prosthetic purposes. Periapical radiography showed that pulp obliteration was severe at the cervical and middle thirds of the root. A cone beam computed tomography (CBCT) was requested in order to assess the level of obliteration. A three-dimensional (3D) template was planned with the CBCT's images along with intraoral scanning. A prototyped guide for guided endodontic access was performed, and the dental procedures were concluded safely. Guided endodontic access was paramount to overcome the pulp obliteration and ensured the endodontic treatment, and prosthetic rehabilitation could be performed.
\end{abstract}

Keywords: Contracted Access Cavity; Endodontics; Guided Endodontics; Pulp Canal Obliteration; Root Canal Treatment

\section{Introduction}

Pulp canal obliteration (PCO), also known as calcific metamorphosis, is characterized by the deposition of calcified tissue along the canal walls. Dental trauma, carious lesions, abfraction, abrasion, pulp capping, malocclusion, orthodontic therapy, parafunctional habits, and aging are factors that can trigger PCO [1,2]. The root canal location and exploration are significantly complicated in teeth with PCO. The difficulty is determined by the root canal anatomy, the extension of calcification, and tooth position in the mouth [3].

Endodontic treatment of teeth with pulp obliteration is a challenging task even for the most experienced endodontists. The use of a dental microscope, ultrasonic tips [4], digital radiographs with different angulations, and cone beam computed tomography (CBCT) [5] provide reliable information to identify obliterated root canals. Despite that, access cavity preparation for such cases is associated with a high failure rate and limited prognosis. Procedural errors can lead to a substantial loss of dentin structure, root canal deviation, or even perforation [6].

Recently, clinical cases and in vitro studies of guided endodontics have been reported using a static computed tomographicbased stereolithographic drill guide system used for access preparation [7-9]. The results are promising, showing that it is a clinically viable method and easy to perform even by the less experienced clinician [10].

This case report aims to describe the guided endodontic access in maxillary left lateral incisor with pulp canal obliteration and coronal tooth structure loss, which required prosthetic rehabilitation. 


\section{Case Report}

A 73-year-old male was referred for dental treatment to the Dental School at Centro Universitário Newton Paiva, Belo Horizonte, Brazil. The patient's medical history revealed hypertension under control with the use of medication and periodic medical check-ups. He had no allergies or other systemic diseases.

Clinical examination revealed dental attrition associated with abfraction lesions in all teeth, compatible with bruxism. The maxillary left lateral incisor had a coronal fracture, and consequently, endodontic treatment was needed due to prosthetic treatment. A periapical radiograph revealed severe pulp canal calcification extending from the cervical to the middle third (Figure 1). There was no radiographic evidence of apical periodontitis. The cavity access was performed with a high-speed diamond round bur. The attempts to locate the canal entrance were unsuccessful. The patient was informed about the complexity of the treatment. After considering the risks and benefits, guided endodontics was elected as the most appropriate treatment approach. Endodontic treatment was started after the patient signed an informed consent form.

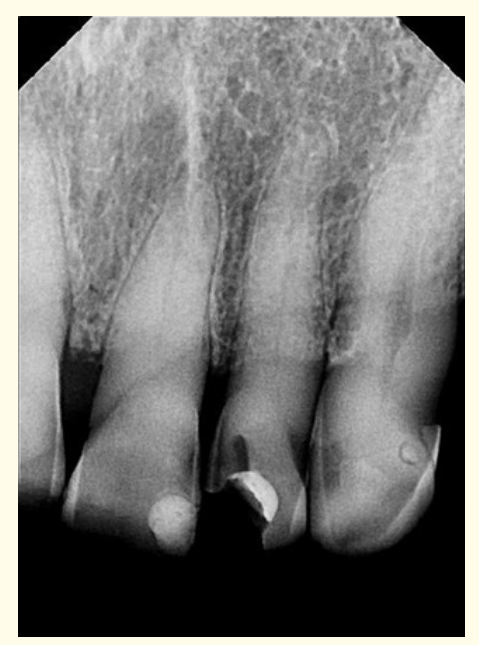

Figure 1: Preoperative radiograph showing the wrong direction of the initial access and pulp calcification.

CBCT was performed using the following settings: 0.2-mm voxel, greyscale, 14 bits, 26.9-second X-ray exposure, $120 \mathrm{kV}$, and 37 mA (iCAT; Imaging Sciences International, Hatfield, PA, USA), which confirmed the severe calcification. The full maxillary arch was scanned (3 Shape Trios 3-Color Intraoral Scanner; Holmens Kanal, Copenhagen, Denmark). The CBCT and teeth scans were aligned and processed using SimPlant (Version 11; Materialise Dental, Leuven, Belgium). A virtual copy of a drill with a diameter of $1.3 \mathrm{~mm}$ and a length of $20 \mathrm{~mm}$ (Neodent Drill for Temp Implants, Ref: 103179; JJGC Ind e Comércio de Materiais Dentarios SA, Curitiba, Brazil) was superimposed onto the scans in a position that allowed the drill access to the entire length of the root. The posi- tion of the drill was confirmed in 3 dimensions. Subsequently, the 3D template was exported as an STL file and sent to a 3D printer (Formlabs2, Formlab Inc., 35 Medford St. Suite 201, Somerville, MA 02143, USA) (Figure 2).

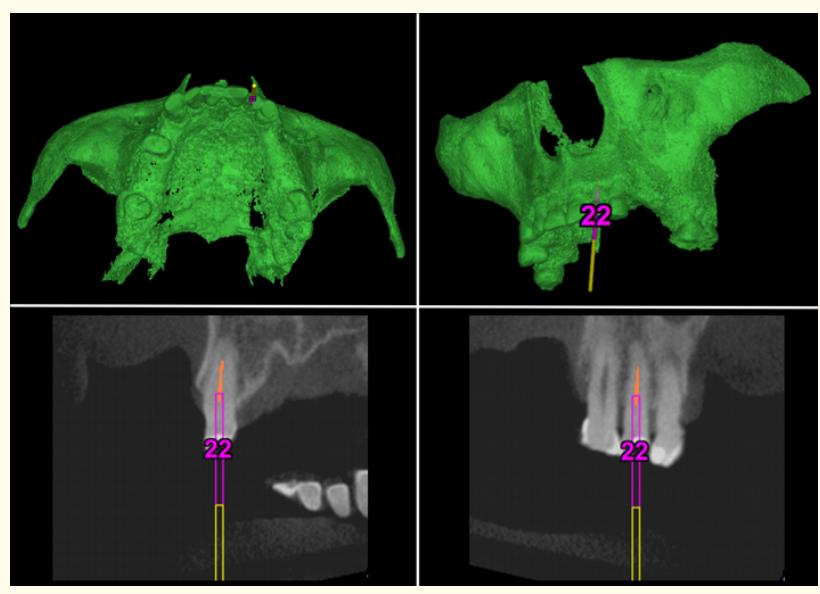

Figure 2: 3D virtual planning.

After checking the adjustment of the template, the access was made with a handpiece set to $950 \mathrm{RPM}$ and $4.1 \mathrm{~N}-\mathrm{cm}$ torque powered by an electric motor VDW Silver (VDW GmbH, Munique, Germany). Saline solution was used to irrigate the bur and prevent microcracks. Intraoperative radiographs were taken with different angulations to check the correct bur position. The canal entrance was located, stainless steel K-files \#10 and \#15 (Dentsply Maillefer, Ballaigues, Switzerland) were used to establish the glide path, the working length (WL) was determined using an apex locator (Root ZX II, JMORITA, USA) and confirmed radiographically (Figures 3 and 4).

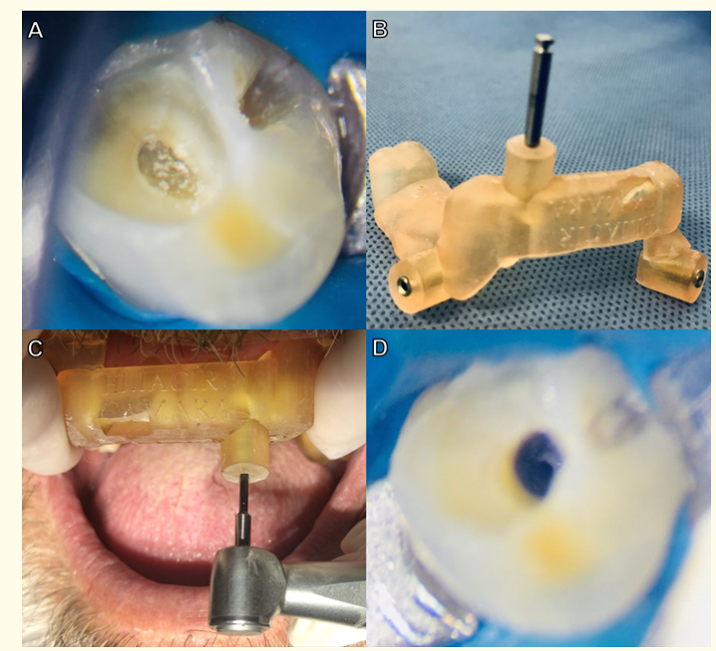

Figure 3: (A) Access performed before virtual planning. (B) Printed guide with bur placed. (C)Initial drilling through pulp calcification. (D) Guided access performed. 


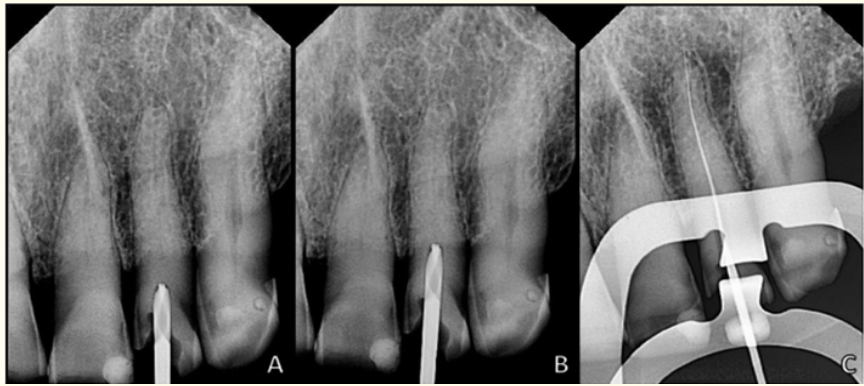

Figure 4: (A) Radiograph at the beginning of the drilling. (B) Radiograph at the end of the drilling. (C) Radiograph confirming the working length.

The tooth was instrumented sequentially with the files 15.06 , 25.04, and 30.04, iRaCe System (FKG Dentaire, Chaux-de-Fonds, Switzerland) up to the WL. Irrigation with $3 \mathrm{~mL}$ of $5.25 \% \mathrm{NaOCl}$ solution was performed after each instrument using a 30-gauge needle attached to a sterile disposable plastic syringe. Upon completion of cleaning and shaping, the root canals were irrigated with $3 \mathrm{~mL}$ of $17 \%$ EDTA solution ( $\mathrm{pH} 7.4$ ) for three minutes and sequentially with $3 \mathrm{~mL}$ of $5.25 \% \mathrm{NaOCl}$. Irrigation was agitated with the XP-endo Finisher (size \#25, taper .00) (FKG Dentaire, Chaux-deFonds, Switzerland) inserted up to WL and activated for $60 \mathrm{~s}$ on rotation mode at $800 \mathrm{RPM}$ and $1 \mathrm{~N}-\mathrm{cm}$ torque with a slow and gentle lengthwise up-and-down movements of 7-10 mm to contact the entire length of the canal. The root canal was dried with sterile paper points and sealed with FM extra length gutta-percha cones (Odous De Deus, Belo Horizonte, MG, Brazil) $1 \mathrm{~mm}$ short of the WL by warm vertical compaction and Pulp Canal Sealer EWT (SybronEndo, Orange, CA, USA). The access cavity was cleaned and sealed with glass ionomer cement (Vitremer, 3M ESPE, Seefeld, Germany) (Figure 5).

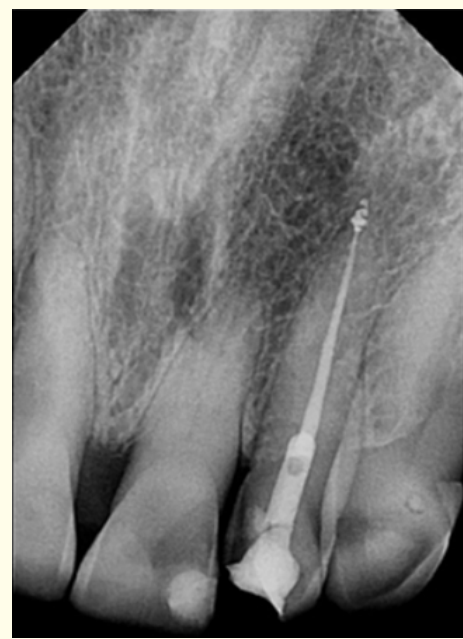

Figure 5: Final radiograph.
At the second appointment, two weeks later, a post space was prepared using a double tapered conical drill for fiberglass post (Exacto \#0.5, Angelus, Londrina, PR, Brazil). The fiber post was cleaned using phosphoric acid gel $(37 \%, 15 \mathrm{~s})$, followed by a silane coupling agent (Silano, Angelus, Londrina, PR, Brazil) application for 1 minute (11). The post was luted with self-adhesive resin cement (RelyX U200; 3M ESPE, St Paul, MN). After 5 minutes, the resin cement was photoactivated on each surface for 60 seconds with a light-emitting diode unit (Radii-Cal; SDI, Bayswater, Australia). The remaining coronal tooth was etched using $37 \%$ phosphoric acid (Cond AC 37; FGM, Joinville, SC, Brazil) for 15 seconds, and the universal adhesive system (Single Bond Universal, 3M ESPE, St Paul, $\mathrm{MN}$ ) was used. The coronary access was built incrementally using Z350 XT composite resin (3M ESPE, St Paul, MN), light activating each increment for 40 seconds until the incisal edge (Figure 6).

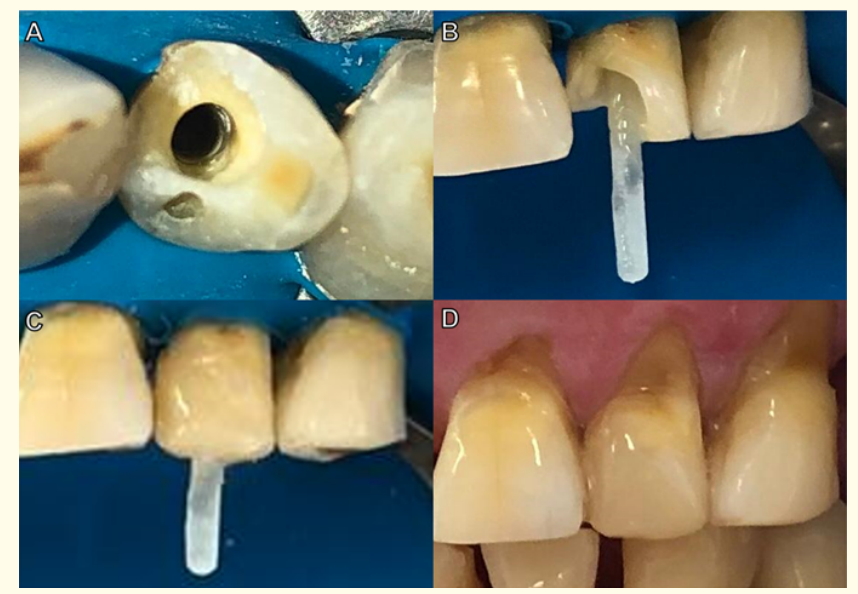

Figure 6: (A) Post space prepared. (B) Fiber post selected according to root canal diameter. (C) Coronal restoration.

(D) Final composite restoration.

After 12 months, the patient returned for clinical and radiographic evaluation (Figure 7). The tooth was asymptomatic; there was no pain to vertical/horizontal percussion or palpation.

\section{Discussion}

The accuracy of guided access preparation seems to be reliable, as reported on in vitro studies. Buchgreitz., et al. [7] reported an average deviation of $0.46 \mathrm{~mm}$ of the tip of the bur. Zehnder., et al. [12] reported a mean angle deviation of $1.81^{\circ}$, with a mean mesial/distal deviation at the tip of the bur of $0.29 \mathrm{~mm}$, buccal/oral of $0.47 \mathrm{~mm}$, and apical/coronal of $0.17 \mathrm{~mm}$. Connert., et al. [13] proposed a new concept of micro guided endodontics, with a mean angle deviation of $1.59^{\circ}$, a mean mesial/distal deviation at the tip of the bur of $0.14 \mathrm{~mm}$, buccal/oral of $0.34 \mathrm{~mm}$ and apical/coronal of $0.12 \mathrm{~mm}$. In this case report, a $1.3 \mathrm{~mm}$ diameter bur was used. This bur diameter is considered ample for mandibular incisors and pos- 


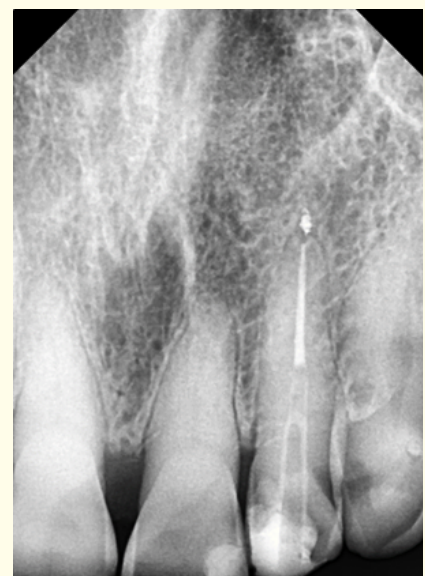

Figure 7: Follow-up 12 months.

terior teeth, however, deviation and wear are considered minimal for maxillary incisors.

Although useful resources such as digital radiographs at different angulations, ultrasonic tips, and magnification, access to teeth with pulp obliteration remain challenging [4], even with computed tomography images, several radiographs are needed during treatment to avoid iatrogenesis. During the clinical procedure, the number of radiographs was significantly reduced, consequently with less exposure to radiation.

During clinical practice, perform access to teeth with pulp obliteration is often associated with a high risk of iatrogenic errors, which can be stressful for the dentists, even for experienced endodontists. Connert., et al. [10] compared a guided endodontic procedure with conventional access preparation using three operators: a 9-year experienced endodontist, a 3-year experienced general dentist, and a newly graduated dentist. Results showed that the mean substance loss was lower for the guided technique than the conventional approach by all operators and reduced treatment duration.

Although the technique requires a very detailed virtual planning, its execution is relatively quick and safe, providing minimal wear towards the root canal.

\section{Conclusion}

The guided endodontic technique in maxillary lateral incisor proved to be a fast, safe, and predictable therapy and can be regarded as an option for the location of calcified root canals, preventing failures in complex cases.

\section{Acknowledgements}

This study was financed in part by the Coordenação de Aperfeiçoamento de Pessoal de Nível Superior Brasil (CAPES) - Finance Code 001.

\section{Conflict of Interest}

The authors deny any conflicts of interest related to this study.

\section{Bibliography}

1. Oginni, Adeleke 0., et al. "Evaluation of radiographs, clinical signs and symptoms associated with pulp canal obliteration: an aid to treatment decision". Dental Traumatology 25.6 (2009): 620-625.

2. Bastos Juliana Vilela and Maria Ilma de Souza Côrtes. "Pulp canal obliteration after traumatic injuries in permanent teethscientific fact or fiction?" Brazilian Oral Research 32 (2018).

3. Robertson Agneta., et al. "Incidence of pulp necrosis subsequent to pulp canal obliteration from trauma of permanent incisors". Journal of Endodontics 22.10 (1996): 557-560.

4. McCabe PS and Paul Michael Howell Dummer. "Pulp canal obliteration: an endodontic diagnosis and treatment challenge". International Endodontic Journal 45.2 (2012): 177-197.

5. Patel S., et al. "Cone beam computed tomography in Endodontics-a review of the literature". International Endodontic Journal 52.8 (2019): 1138-1152.

6. Lang H., et al. "Impact of endodontic treatments on the rigidity of the root". Journal of Dental Research 85.4 (2006): 364-368.

7. Buchgreitz J., et al. "Guided access cavity preparation using cone-beam computed tomography and optical surface scans-an ex vivo study". International Endodontic Journal 49.8 (2016): 790-795.

8. Lara-Mendes Sônia TO., et al. "A new approach for minimally invasive access to severely calcified anterior teeth using the guided endodontics technique". Journal of Endodontics 44.10 (2018): 1578-1582.

9. Buchgreitz., et al. "Guided Endodontics Modified for Treating Molars by Using an Intracoronal Guide Technique". Journal of Endodontics 45.6 (2019): 818-823.

10. Connert Thomas., et al. "Guided Endodontics versus Conventional Access Cavity Preparation: A Comparative Study on Substance Loss Using 3-dimensional-printed Teeth". Journal of Endodontics 45.3 (2019): 327-331. 
11. de Sousa Menezes Murilo, et al. "Fiber post etching with hydrogen peroxide: effect of concentration and application time". Journal of Endodontics 37.3 (2011): 398-402.

12. Zehnder MS., et al. "Guided endodontics: accuracy of a novel method for guided access cavity preparation and root canal location". International Endodontic Journal 49.10 (2016): 966972.

13. Connert Thomas., et al. "Microguided endodontics: accuracy of a miniaturized technique for apically extended access cavity preparation in anterior teeth". Journal of Endodontics 43.5 (2017): 787-790.

\section{Assets from publication with us}

- Prompt Acknowledgement after receiving the article

- Thorough Double blinded peer review

- Rapid Publication

- Issue of Publication Certificate

- High visibility of your Published work

Website: www.actascientific.com/

Submit Article: www.actascientific.com/submission.php

Email us: editor@actascientific.com

Contact us: +919182824667 